\title{
Three-dimensional virtual model surgery to fabricate the digital intermediate splint
}

\author{
Sun, Yi ; Luebbers, Heinz-Theo ; Politis, Constantinus
}

\begin{abstract}
Model surgery is the classical requirement for fabricating an intermediate splint for bimaxillary surgery. However, several sources of error complicate the conventional manual technique for making an intermediate splint, and it can be difficult to reposition the cast model of the upper jaw in the desired position. Additionally, the manual procedure is time consuming and requires a lot of laboratory work. Here, we present a technical note on virtual model surgery, a computer-assisted method that combines 3-dimensional cone beam computed tomography (CBCT) of the articulator and an optical scan of the plaster cast models. A registration block is designed to match the detailed occlusal surface scan to the CBCT data. A paired-point-based registration algorithm is utilized to calculate the registration metric and to transfer the scanned plaster cast model of the upper jaw to the desired position. The detailed protocol is described, and one clinical case is presented.
\end{abstract}

DOI: https://doi.org/10.1097/SCS.0b013e3182700e4f

Posted at the Zurich Open Repository and Archive, University of Zurich

ZORA URL: https://doi.org/10.5167/uzh-86304

Journal Article

Accepted Version

Originally published at:

Sun, Yi; Luebbers, Heinz-Theo; Politis, Constantinus (2013). Three-dimensional virtual model surgery to fabricate the digital intermediate splint. Journal of Craniofacial Surgery, 24(2):563-565.

DOI: https://doi.org/10.1097/SCS.0b013e3182700e4f 
Title: Three-dimensional virtual model surgery to fabricate the digital intermediate splint:

technical note

Yi Sun, MSc ${ }^{1,2}$, Heinz-Theo Luebbers MD DMD ${ }^{3}$, Constantinus Politis, MD DDS ${ }^{1,2 \S}$

${ }^{1}$ Oral and Maxillofacial Surgery, St. John's Hospital, Schiepse bos 6, 3600 Genk,

Belgium. (Head: C. Politis, Prof. MD. DDS )

${ }^{2}$ Faculty of Medicine, Hasselt University, Diepenbeek, Belgium.

${ }^{3}$ Clinic for Cranio-Maxillofacial Surgery, University Hospital of Zurich. Switzerland.

${ }^{\S}$ Corresponding author

Prof. Dr. C. Politis

Department of Oral and Maxillofacial Surgery

St. Johan's Hospital

Schiepse Bos 6

3600 Genk

Belgium

Tel: +3289326161

Fax: +32893217 79

Email: constantinus.politis@uhasselt.be

\section{Conflicts of Interest and Source of Funding:}

None 


\begin{abstract}
Model surgery is the classical requirement for fabricating an intermediate splint for bimaxillary surgery. However, several sources of error complicate the conventional manual technique for making an intermediate splint, and it can be difficult to reposition the cast model of the upper jaw in the desired position. Additionally, the manual procedure is time consuming and requires a lot of laboratory work. Here we present a technical note on virtual model surgery, a computer-assisted method that combines three-dimensional cone beam computed tomography (CBCT) of the articulator and an optical scan of the plaster cast models. A registration block is designed to match the detailed occlusal surface scan to the CBCT data. A pairedpoint-based registration algorithm is utilized to calculate the registration metric and to transfer the scanned plaster cast model of the upper jaw to the desired position. The detailed protocol is described, and one clinical case is presented.
\end{abstract}

Key words: bimaxillary surgery; computer assisted; intermediate splint; 3D printer 


\section{Introduction}

Orthognathic surgery is meant to correct conditions of the jaw and face related to structure, growth, sleep apnea, tempromandibular joint disorders, malocclusion problems owing to skeletal disharmonies, or other orthodontic problems that cannot be treated with orthodontics only. In orthognatic surgery, there is a growing tendency for bimaxillary surgery because bimaxillary surgery usually decreases the amount of movement in each single jaw, rendering the result more stable. It also allows for better aesthetics. Given the growing concern about achieving a pre-operative stable occlusion, many centers opt for multisegmental osteotomies in the upper jaw together with a bimaxillary approach, thus minimizing the amount of orthodontic movements that carry a risk of relapse..$^{1}$

To our knowledge there is no valid data regarding the number of orthognathic surgical procedure in Western Europe. For the United States of America based on the data (1991 to 2009) out of training programs we calculate about 50’000 procedures per year. From our clinical experience we expect $30^{\prime} 000$ to $40^{\prime} 000$ to be bimaxillary procedures in order to achieve correct occlusion and optimal facial aesthetics. A routine procedure consists of the following three steps to prepare for bimaxillary surgery: bite and facebow registration of the patient, transfer of the registration to the plaster models in the articulator, and model surgery to fabricate the intermediate splint. There are several sources of error during model surgery. .2 For example, measurement of the positions of the reference points is not accurate, and it is difficult to position the plaster cast model on the exact position delineated by the surgical 
treatment plan. Furthermore, during fabrication of the intermediate splint, the splint may not fit the cast models perfectly.

Over the past twenty years, various instruments and methods have been developed to minimize the errors associated with model surgery.,$\underline{3,4,5,6,7, \underline{8}}$ Additionally, the accuracy of model surgery depends on the kind of articulator used for the model surgery. $\cdot,-\frac{910}{}$ Bamber et al. ${ }^{\stackrel{9}{ }}$ reported that the Eastman technique was relatively more accurate than the Lockwood keyspacer, especially in the vertical plane. However, we still lack sufficient control of the rotation and translation of the plaster cast models during model surgery.

With the development of computer-aided surgery, a three-dimensional (3D) virtual program (3Txer, version 1.0, Orapix, Seoul, Korea) has replaced the manual work of model surgery. $\frac{11}{}$ This program allows the surgeon to mount the scanned plaster cast models, which are in the central relationship, into the virtual articulator. Afterward, the digitized upper jaw can be virtually placed into the desired position, and an intermediate splint is generated and printed out via stereolithography. Although this virtual model surgery seems to be more reliable and accurate than manual model surgery, this method is limited by the requirement of measuring the $3 \mathrm{D}$ coordinates of each tooth's position and transferring it to the virtual articulator. Furthermore, the accuracy of stereolithographic intermediate splint fabrication needs to be improved. Here we present a new workflow to perform virtual model surgery, and we discuss a relevant clinical case. 


\section{Materials and Methods}

A Skyview cone beam computed tomography (CBCT; Cefla Dental, Imola, Italy) scanner was used in our study. The scanning parameters were 9 inch, dentition mode. Each slice was of composed $512 \times 512$ pixels and the voxel size was $0.3 \times 0.3 \times 0.3 \mathrm{~mm}$.

Wax-bite registration was taken in the centric relation to record the anatomical relationship of both jaws in passive centric occlusion. Facebow registration was subsequently performed, and the patient coordinate system was transferred to the Dentatus articulator system (Fig. 1A), type ARH (Dentatus AB, Spanga, Sweden).

Plaster cast models were scanned with an Activity 102 dental scanner (Smart optics Sensortechnik GmbH, Bochum, Germany). In the first step, the 3D optical scan process consisted of separate scans of the mandible and maxilla models. Second, the plaster cast models of the mandible and maxilla were mounted in an Adesso Split magnetic articulator (Fig. 1B) in passive centric occlusion (Baumann Dental, KelternEllmendingen, Germany). This magnetic articulator was placed in the dental scanner, and a vestibular scan of both plaster cast models (maxilla and mandible) was performed. Third, both virtual models from the first step were registered to the result from the second step via surface matching. Output data were saved in Standard Tessellation Language (STL) format and had a recurrence accuracy of $100 \mu \mathrm{m}$.

The data from the previous step were exported to VisCAM Mesh 5.0 (Marcam Engineering $\mathrm{GmbH}$, Bremen,Germany; Fig. 1C). A virtual wax bite was generated by filling the gap between the virtual upper and lower jaws. A pre-designed universal 
virtual registration frame was assembled to this virtual wax bite. We designated this computer-designed object (the virtual wax bite and the virtual registration frame together) as a virtual registration block (CAD_R; Fig. 1D).

CAD_R was printed via an Alaris 3D printer (Objet Ltd, Israel; Fig. 1E). The layer thickness of this printed model was 0.0011 inches, which ensured smooth, accurate, and highly detailed representation of the design. This printed model was placed back in the Adesso Split magnetic articulator for a CBCT scan together with the plaster cast model of the upper and lower jaws. The DICOM (Digital Image Communications in Medicine) files were imported into the Amira medical imaging software 5.2.2 (Visage Imaging $\mathrm{GmbH}$, Berlin, Germany) for further processing.

Surface rendering was performed on the CBCT data (Fig. 2A). Surface areas of the four cylinders in the CAD_R and CBCT data were selected as the reference area for surface registration. A detailed occlusal surface $(100 \mu \mathrm{m})$ was therefore registered to and integrated with the $\mathrm{CBCT}$ data (Figs. 2B, C).

The top level of the Adesso Split articulator was set as the Frankfort horizontal (FH) plane (Fig. 3A). The midsagittal plane was set perpendicular to the FH plane and passed through the middle of the vertical axis of the Adesso Split articulator (Fig. 3B). The FH plane was set as $\mathrm{X}-\mathrm{Y}$ plane and the midsagittal plane is set as $\mathrm{Y}-\mathrm{Z}$ plane. After setting the CBCT data in the correct coordinate system, virtual model surgery was performed according to the pre-operative surgical plan that was based on a clinical exam, a cast model study, and two-dimensional cephalometry tracing. In order to place the virtual upper jaw in the correct position, three points were virtually 
indicated: the contact point of the central incisor and the mesiobuccal crown tips of teeth 16 and 26 (Fig. 3C). The corresponding 3D coordinates of these three points were recorded and the final 3D coordinate of each points were fed into the Amira medical imaging software 5.2.2 (Visage Imaging GmbH, Berlin, Germany) according to the pre-operative surgical plan. Paired-point-based registration was performed; the registration metric was calculated automatically and applied to the virtual upper jaw, transferring the virtual upper jaw to the required position (Fig. 3D). The final position of the maxilla and the initial position of the mandible with detailed occlusal information were exported to VisCAM Mesh 5.0 (Marcam Engineering $\mathrm{GmbH}$, Bremen,Germany) in STL format. An intermediate splint was designed in this CAD tool and printed with the Objet Alaris 3D printer.

\section{Results}

One 32-year-old female patient underwent bimaxillary surgery. The preoperative surgical plan was for LeFort I advancement by $4 \mathrm{~mm}$, with $6 \mathrm{~mm}$ intrusion of the anterior part of the maxilla and subsequent bilateral sagittal split osteotomy advancement. A customized CAD_R model was designed and printed, and a digital intermediate splint was fabricated according to the protocol in Materials and Methods. During surgery, this intermediate splint was used to position the maxilla in the planned position (Fig. 4). The feedback from the surgeon was that the fit of the printed intermediate splint was better than that of conventional intermediate splints. Immediate post-operative CBCT was carried out and registered to the pre-operative 
CBCT data (Fig. 5). We checked the post-operative outcome: the advancement of the maxilla was $4.6 \mathrm{~mm}$ and the intrusion of anterior part of the maxilla was $6.8 \mathrm{~mm}$.

\section{Discussion}

Here we have presented a protocol that combines optical scan data and CBCT data to fabricate an intermediate splint for bimaxillary orthognatic surgery. The advantages of this method are that the registration block is helpful to transfer the detailed occlusal data to the $\mathrm{CBCT}$ data, and that the three paired-point-based registration algorithm allows automatic surgical movement of the maxilla as long as the initial and final 3D coordinates of each reference points are known by the computer. However, the paired-point-based registration algorithm was only able to achieve the best matching position. To overcome this problem, the Amira software also provides the option to manipulate the virtual upper jaw in $3 \mathrm{D}$ to define the rotation center and the rotation axis. A third advantage of this workflow is that it could be used to simulate various surgical plans in 3D, assisting the surgeon in selecting the best treatment plan.

On the other hand, we perceive two limitations of this method. First, the FH plane and the midsagittal plane are controlled virtually by the surgeon via computer, which may introduce errors that are transferred to the fabrication of the intermediate splint. Second, this method only replaces conventional model surgery; bite registration, facebow registration, and transferring data from the registrations to the articulator are still necessary, and the errors involved in these steps would persist in 
our method.

Approximately 60 minutes are required to scan the cast model in centric relation and design the registration block in the CAD software. It takes another 60 minutes for surgical planning and fabrication of the virtual intermediate splint in the CAD software. Printing the intermediate splint can be accomplished within 2 hours via the Alaris $3 \mathrm{D}$ printer. However, the presented clinical case revealed that in our hands, and compared to conventional model surgery, the computer-assisted method was less time consuming and led to acceptable surgical accuracy.

Since we have only presented one case report, a larger set of clinical cases should be assessed to validate the reliability of this procedure. Furthermore, in the future the workflow should integrate a digital procedure to set the FH plane directly in accordance with patient anatomy. This advancement will limit the error introduced by facebow registration and consecutive transfer to the articulator.

\section{Acknowledgements}

The authors thank Prof. J. A. Obwegeser, University Hospital Zürich, Switzerland for information regarding the number of orthognathic surgical procedures in the United States of America. 
1. Kretschmer WB, Baciut G, Baciut M, et al. Transverse stability of 3-piece Le Fort I osteotomies. J Oral Maxillofac Surg 2011;69:861-869

2. Olszewski R, Reychler H. [Limitations of orthognathic model surgery: theoretical and practical implications]. Rev Stomatol Chir Maxillofac 2004;105:165-169

3. Akram A, Collin J, Postlethwaite K, et al. Planned to perfection? Use of the model repositioning instrument to plan maxillary movements for orthognathic surgery. Orthodontics (Chic.) $2011 ; 12: 130-133$

4. Anwar M, Harris M. Model surgery for orthognathic planning. Br J Oral Maxillofac Surg $1990 ; 28: 393-397$

5. Ellis E, 3rd. Accuracy of model surgery: evaluation of an old technique and introduction of a new one. J Oral Maxillofac Surg 1990;48:1161-1167

6. Omura T, Glickman RS, Super S. Method to verify the accuracy of model surgery and prediction tracing. Int J Adult Orthodon Orthognath Surg 1996;11:265-270

7. Schwestka-Polly R, Kubein-Meesenburg D, Luhr HG. Techniques for achieving threedimensional positioning of the maxilla applied in conjunction with the Gottingen concept. Int $J$ Adult Orthodon Orthognath Surg 1998;13:248-258

8. Sharifi A, Jones R, Ayoub A, et al. How accurate is model planning for orthognathic surgery? Int J Oral Maxillofac Surg 2008;37:1089-1093

9. Bamber MA, Harris M, Nacher C. A validation of two orthognathic model surgery techniques. 
10. O'Malley AM, Milosevic A. Comparison of three facebow/semi-adjustable articulator systems for planning orthognathic surgery. Br J Oral Maxillofac Surg 2000;38:185-190

11. Song KG, Baek SH. Comparison of the accuracy of the three-dimensional virtual method and the conventional manual method for model surgery and intermediate wafer fabrication. Oral Surg Oral Med Oral Pathol Oral Radiol Endod 2009;107:13-21 


\section{Figure Legends}
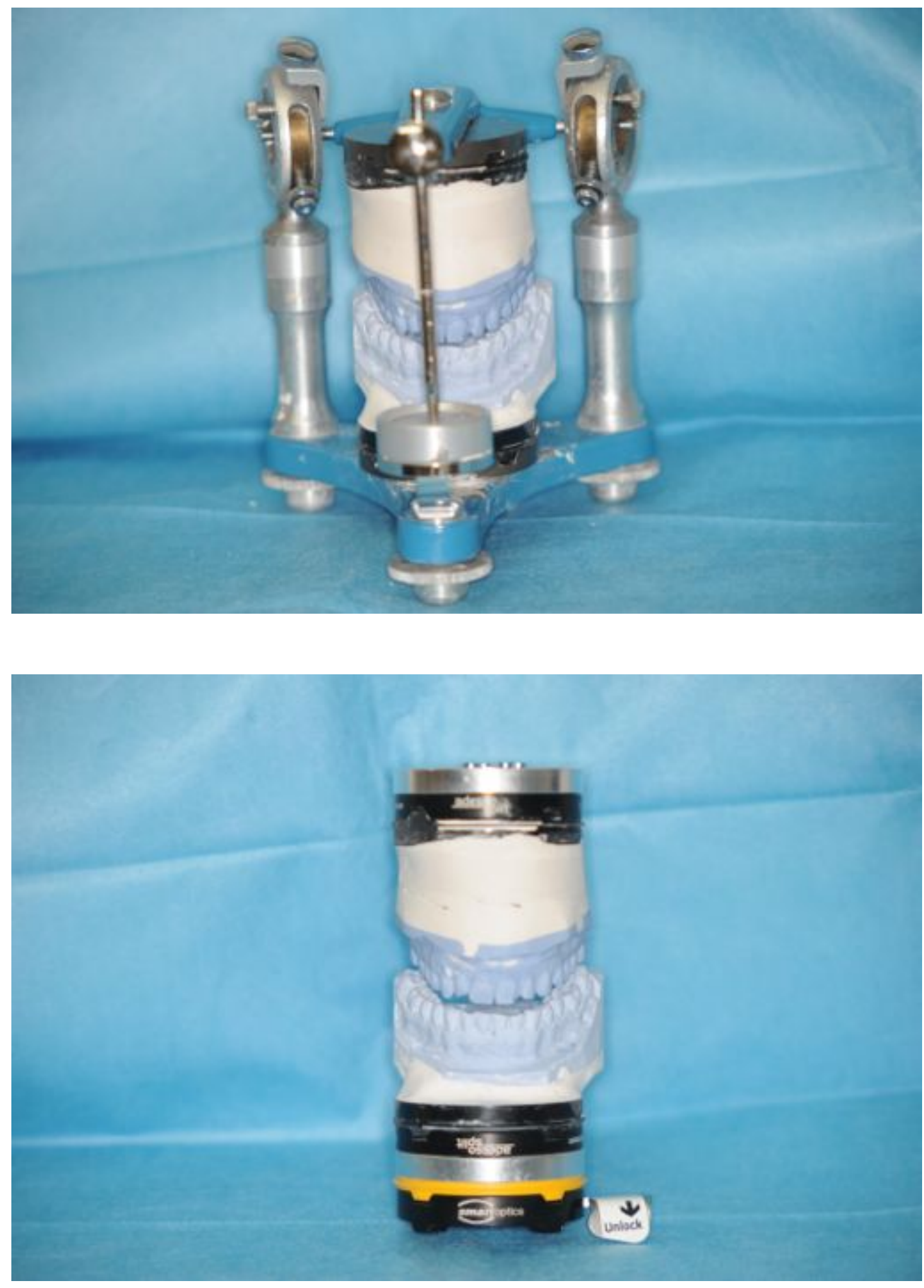

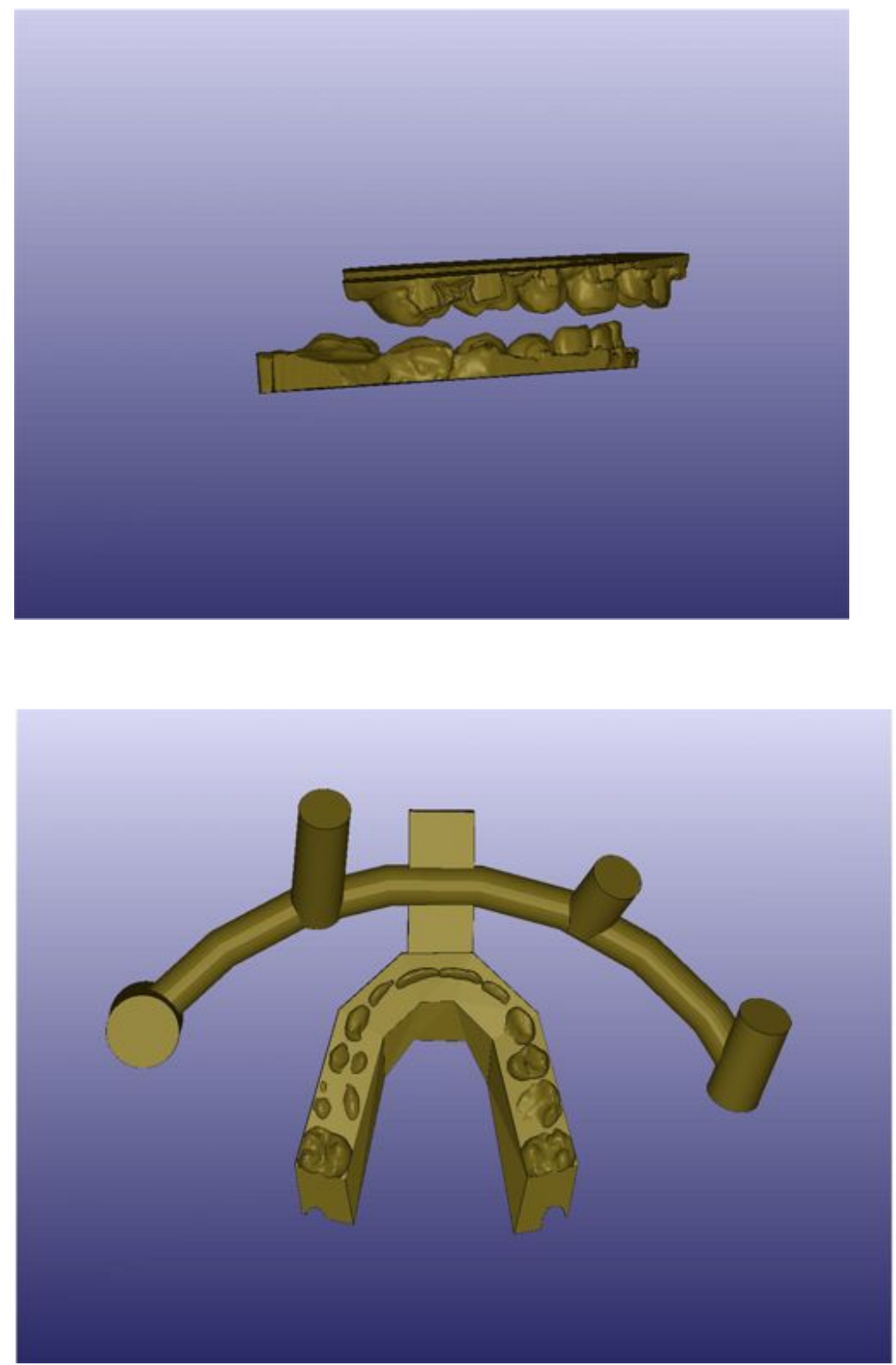


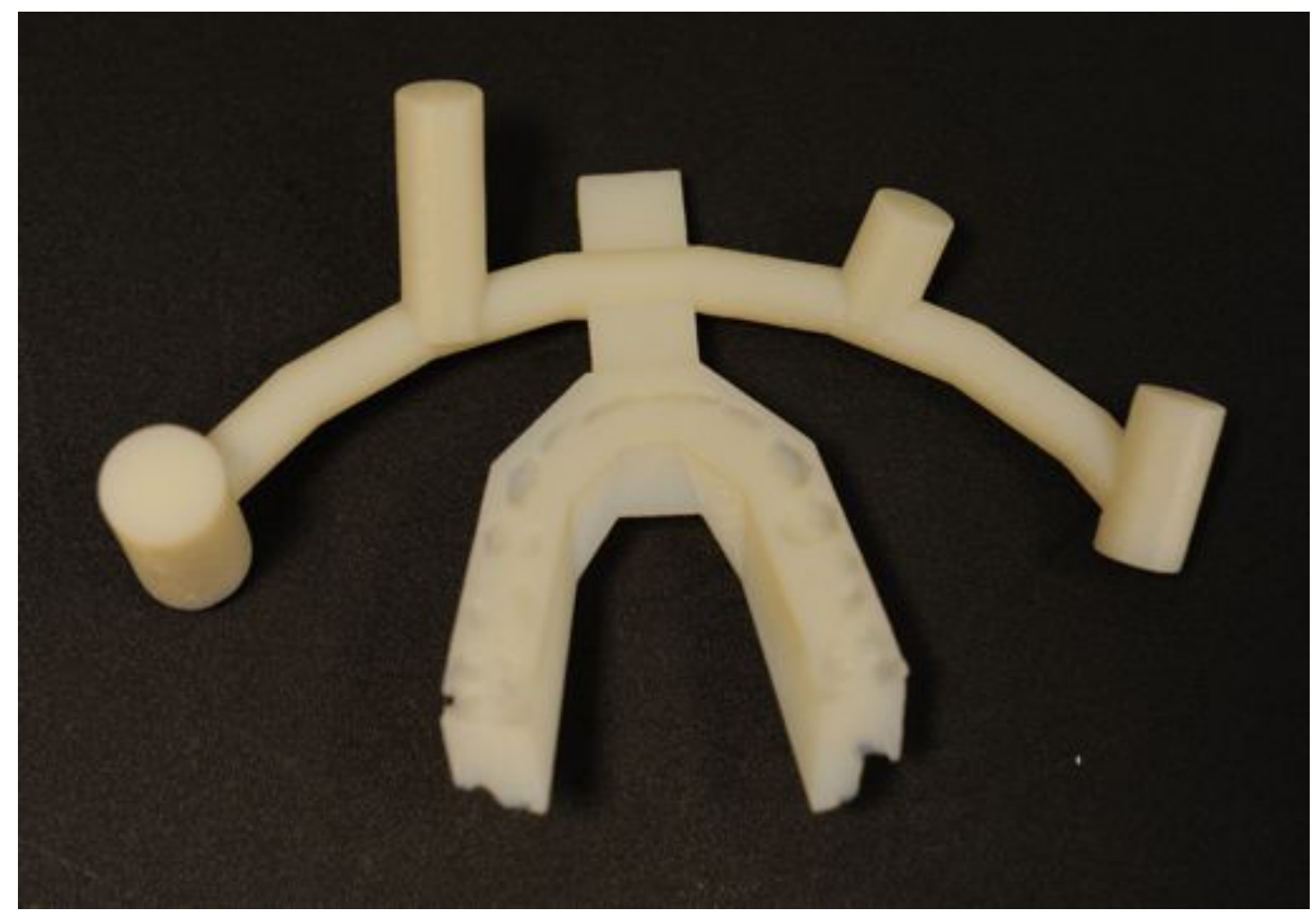

Figure 1. Procedure to design a customized registration block. (A) Plaster cast models are mounted in the Dentatus articulator. (B) Plaster cast models are placed in the Adesso Split articulator for optical scanning. (C) Scanned plaster cast model in centric relation. (D) CAD model of the customized registration block (CAD_R), the surface of the cylinders are used for the surface registration to the CBCT data. (E) Printed CAD_R model by Alaris 3D printer. 

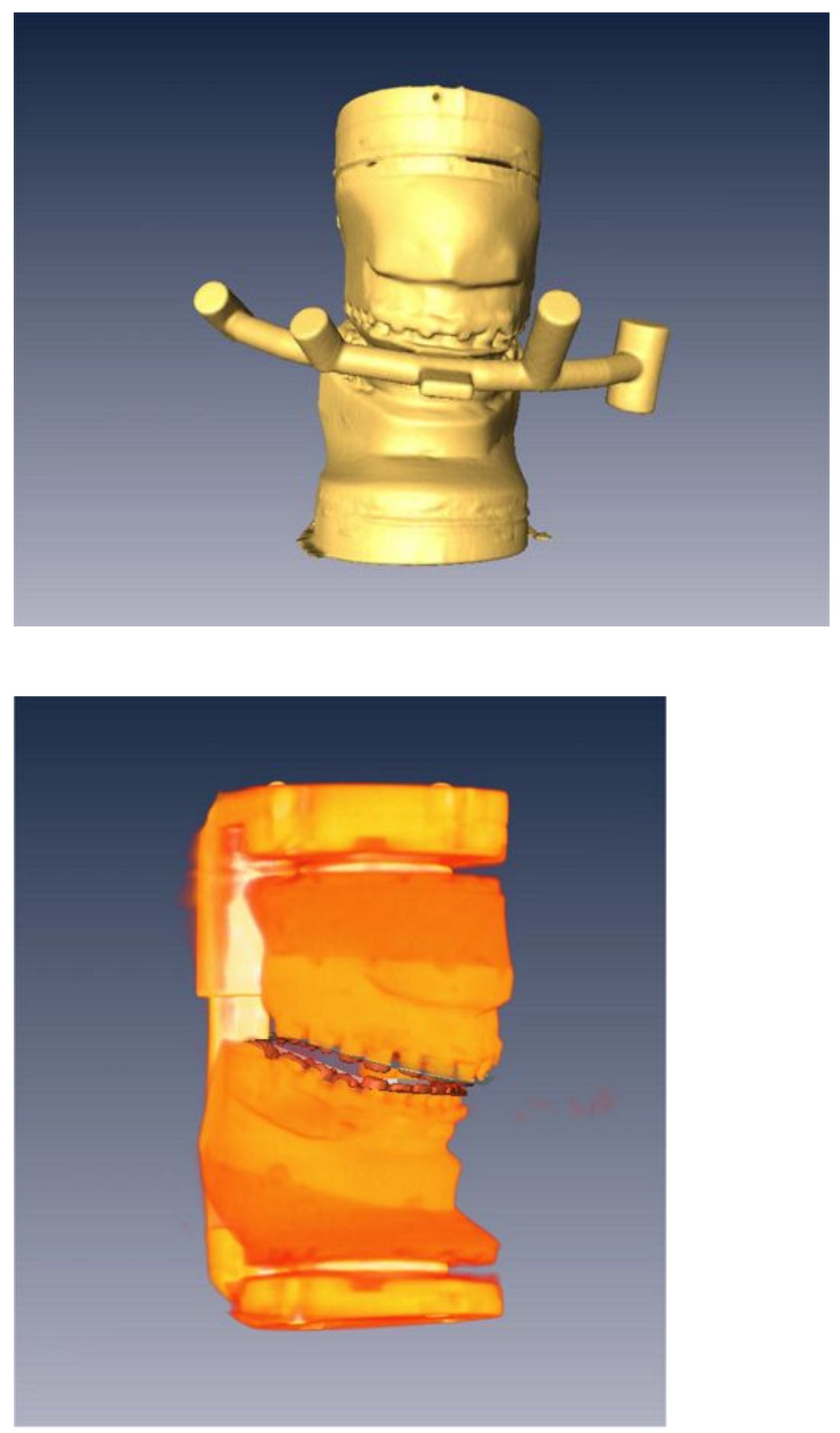

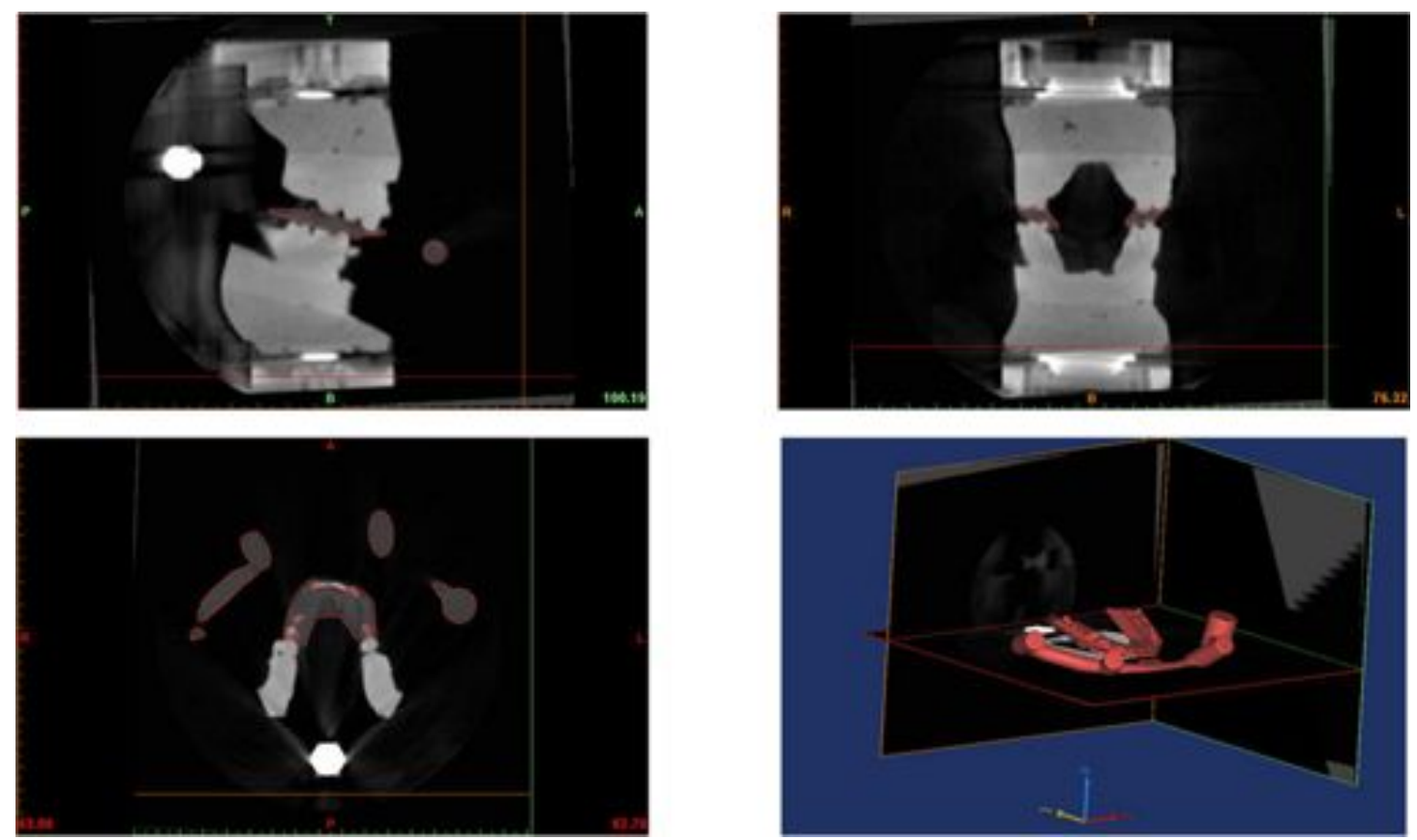

Figure 2. Registration procedure to match the scanned plaster cast model to the CBCT data. (A) Surface rendering of the CBCT data from the customized registration block together with the articulator. (B) CAD_R is registered to the CBCT data according to the surface of four cylinders. (C) Checking the accuracy of the registration. 


[혀



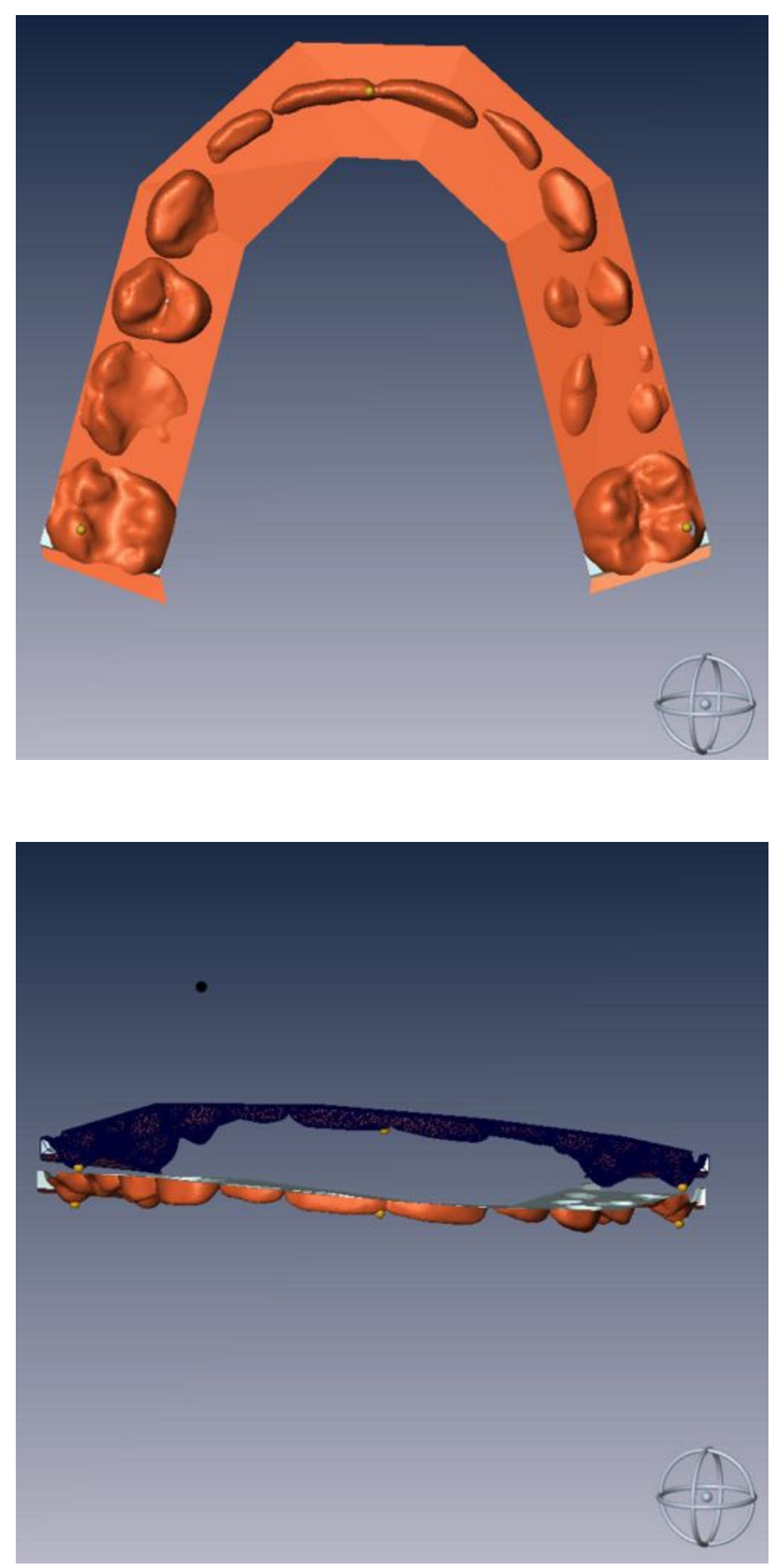

Figure 3. Surgical planning via the Amira software. (A) The top level of the Adesso Split articulator is set as the FH plane. (B) The midsagittal plane is set perpendicular to the FH plane and passes through the vertical axis of the bar of the Adesso Split articulator. (C) Three references points are placed on the virtual upper jaw. (D) The 
virtual upper jaw is moved automatically from the initial position to the desired position. 


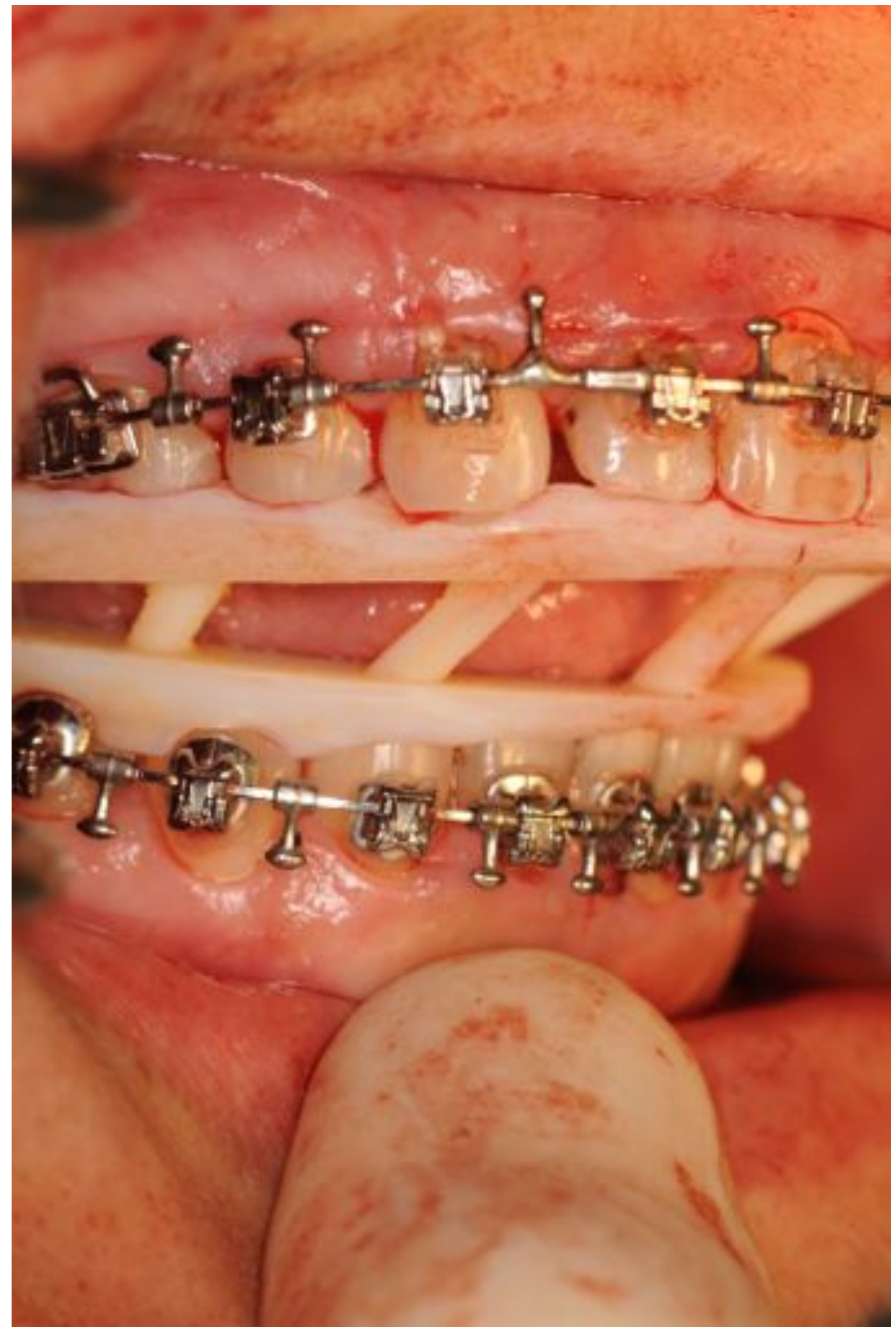

Figure 4. Use of the printed intermediate splint during bimaxillary surgery. 

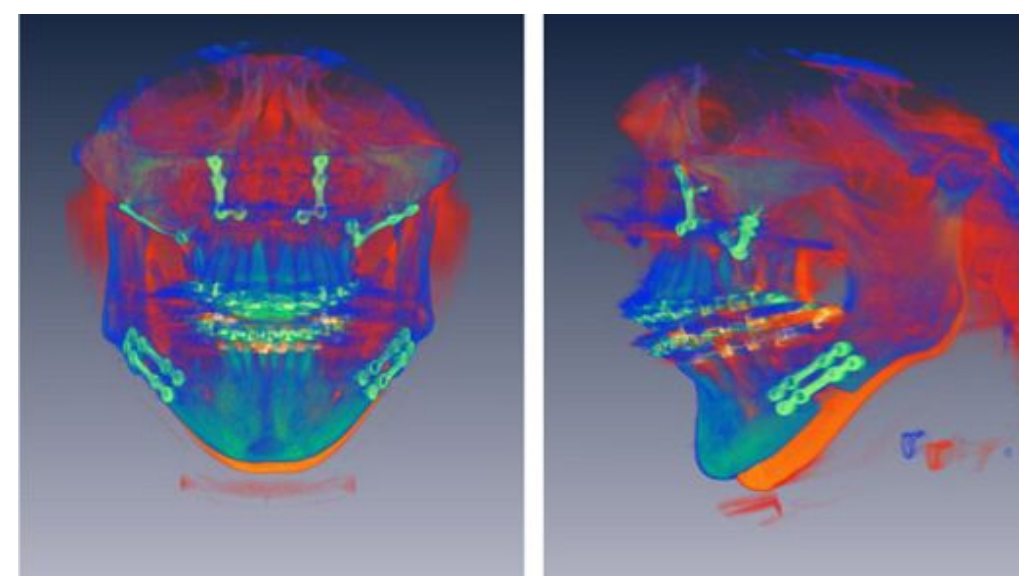

Figure 5. Immediate post-operative $\mathrm{CBCT}$ data is registered to the pre-operative CBCT data to evaluate the surgical change. 